\title{
Alterations in calmodulin content and localization in areas of rat brain after repeated intermittent amphetamine
}

\author{
Margaret E. Gnegy ${ }^{1}$, G.H. Keikilani Hewlett ${ }^{1}$, Susan L. Yee ${ }^{1}$ and Michael J. Welsh ${ }^{2}$ \\ Departments of 'Pharmacology, and '2Anatomy and Cell Biology, The University of Michigan Medical School, Ann Arbor, MI (U.S.A.)
}

(Accepted 21 May 1991)

Key words: Bchavioral scnsitization; Calmodulin translocation; Nucleus accumbens; Striatum; Dopamine

\begin{abstract}
To assess whether calmodulin $(\mathrm{CaM})$ could have a role in the behavioral sensitization induced by repeated intermittent amphetamine, CaM content was determined in several brain areas from rats repeatedly administered saline or amphetamine. Rats were treated with amphetamine using an escalating dose paradigm and withdrawn for either 4 weeks (withdrawn group) or 30 min (non-withdrawn group). CaM content was measured in cytosol and $100,000 \times \mathrm{g}$ membrane fractions from striatum, limbic forebrain. medial prefrontal cortex. hippocampus and cerebellum. In the withdrawn group, CaM was significantly increased in both striatal membranes and cytosol and in the mesolimbic membranes from amphetamine-treated rats. There were no changes in CaM in the medial prefrontal cortex, hippocampus or cerebellum. In the non-withdrawn group, there was no significant change in CaM in striatal or mesolimbic fractions but CaM was significantly decreased in cytosol of the medial prefrontal cortex and hippocampus as compared to saline controls. This decrease could be related to the tolerance that has developed to the amphetamine after the repeated treatments. In the withdrawn group, challenge with a low dose of amphetamine (1 $\mathrm{mg} / \mathrm{kg}$ ) elicited a translocation of CaM from membranes to cytosol in the striatum and limbic forebrain of rats repeatedly treated with amphetamine, but not in saline-treated rats. Our findings that the change in $\mathrm{CaM}$ occurs in striatum and limbic forebrain, requires time after trcatment to develop and exhibits persistence after withdrawal correlate with known characteristics of behavioral sensitization to amphetamine. These results suggest that CaM could contribute to neurochemical events underlying behavioral sensitization to amphetamine.
\end{abstract}

\section{INTRODUCTION}

Repeated use of amphetamine (AMPH) in humans can lead to a psychotic state that clinically resembles paranoid schizophrenia ${ }^{4,7,21}$. AMPH-induced psychosis disappears upon withdrawal from the drug or after treatment with dopamine (DA) receptor blocking drugs. Addicts will retain increased sensitivity to the drug for years after withdrawal from the $\mathrm{drug}^{38}$. Behavioral scnsitization after repeated intermittent AMPH can be demonstrated in animals. The behavioral sensitization in animals is characterized by a more rapid onset of stereotyped behavior and more intense stereotyped movements than in controls and a marked increase in AMPH-induced rotational behavior (for review, see ref. 33). The behavioral effects develop with time after withdrawal from repeated intermittent AMPH treatment and will persist for up to a year ${ }^{28}$. Thus AMPH-induced behavioral sensitization in animals may be due to long-term neural adaptations in areas of brain responsible for the psychomotor or psychotogenic effects of AMPH. Considerable evidence suggests that AMPH-induced stereo- typed behavior and AMPH-induced locomotor behavior are considered due to increased DA release in the caudate putamen and nucleus accumbens, respectively ${ }^{3,17,26}$. Similarly, the activation of DA systems is necessary for the development of sensitization. Administration of DA receptor antagonists, particularly DA D 1 receptor antagonists, with AMPH blocks the development of behavioral sensitization ${ }^{1,22,40}$. Several studies have demonstrated that behavioral sensitization to AMPH is accompanied by an enhanced ability of DA to be released by stimuli, e.g. AMPH, $\mathrm{K}^{+}$or electrical stimulation $^{19.34 .41}$. The increased releasability of DA displays many of the characteristics of behavioral sensitization such as persistence and development upon withdrawal $^{32,33}$

We have investigated changes in the endogenous $\mathrm{Ca}^{2+}$. binding protein, calmodulin ( $\mathrm{CaM})$, in rat striatum following repeated treatment with AMPH. CaM modulates the effect of $\mathrm{Ca}^{2+}$ on cyclic nucleotide metabolism and protein phosphorylation-dephosphorylation, and alters calcium transport and cytoskeletal interactions ${ }^{24}$. Calmodulin is particularly enriched in synaptic fractions of the 
brain $^{10}$ and is involved in synthesis and release of transmitters $^{6,23,24}$. Pharmacological treatments that regulate dopaminergic activity result in changes in $\mathrm{CaM}$ in subcellular fractions of striatum ${ }^{8,13}$.

In previous studies we found that $\mathrm{CaM}$ is increased in rat striatum upon withdrawal from repeated treatment with $\mathrm{AMPH}^{31,37}$. In addition, we found that a challenge with a low dose of AMPH will elicit a translocation of $\mathrm{CaM}$ from membranes to cytosol in striatum from rats that were repeatedly treated with AMPH but not sa$\operatorname{line}^{37}$. This translocation did not occur in rats not sensitized to AMPH. The involvement of CaM in so many neural functions suggests that it could play a role in AMPH-induced behavioral sensitization. To further explore a role for $\mathrm{CaM}$ in behavioral sensitization, we measured $\mathrm{CaM}$ in membrane and cytosol fractions of several rat brain areas after repeated intermittent AMPH. These studies were conducted in one group of rats that were withdrawn 4 weeks from the repeated AMPH treatment and in another group that was not withdrawn following AMPH treatment. We found that CaM was selectively increased in striatum and mesolimbic areas after withdrawal from repeated AMPH. On the other hand, CaM either showed no change or was decreased in several brain areas in the non-withdrawn AMPH group as compared to controls.

\section{MATERIALS AND METHODS}

\section{Chronic treatment paradigm}

Female Sprague-Dawley derived rats, 150-200 g (Holtzman Co. Inc., Madison, WI) were used in all experiments. Rats were divided into two groups; half was injected i.p. with saline (SAL) and the other half with escalating doses of AMPH. Animals were housed in groups of 6 and injections were given twice a day for 5 days with 10-12 h separating the two injections; this was followed by two drug-free days. This schedule was repeated for 4 weeks. The rats received a total of 40 injections ( 20 injection days) according to the following schedule: injection days 1-3 (1.0 mg/kg); 4-5 (2 mg/kg); 6-7 (3 mg/kg); 8-9 (4 $\mathrm{mg} / \mathrm{kg}) ; 10-11$ (5 mg/kg); 12-14 (6 mg/kg); 15-17 (7 mg/kg); 18-20 (8 $\mathrm{mg} / \mathrm{kg}$ ). This escalating dose regimen was similar to that of Robinson and Camp ${ }^{35}$. The control group received an equivalent number of SAL injections. For one experiment, rats were sacrificed $\mathbf{3 0} \mathrm{min}$ after the last injection on the 20th injection day. This was the non-withdrawn group. The withdrawn group was sacrificed 28 days after the last injection. In the withdrawn group, rats received an i.p. challenge injection of either $0.9 \%$ SAL or $1.0 \mathrm{mg} / \mathrm{kg}$ AMPH $30 \mathrm{~min}$ before sacrifice such that 4 groups were formed with 5 rats in each group: SAL-SAL (SS), SAL-AMPH (SA), AMPH-SAL (AS) and AMPH-AMPH (AA). The 4-week treatment with AMPH did not significantly alter the growth rate of the rats. Throughout the study the average weight of the AMPH-treated rats was not significantly different from the average weight of the SAL control groups.

All animals were sacrificed on the same day and the brain areas were dissected on ice, weighed and the tissue frozen in liquid $\mathbf{N}_{2}$. Brain areas were dissected using a brain cutting block as described by Heffner et al. ${ }^{16}$. The mesolimbic area (or limbic forebrain) contains both nucleus accumbens and olfactory tubercle. Medial prefrontal cortex was dissected, which was shown to have the highest concentration of $\mathrm{DA}^{16}$. Tissue was stored for no longer than 2 weeks until assayed for CaM.

\section{Measurement of CaM content}

Tissue was prepared for measurement of $\mathrm{CaM}$ by homogenization in $40 \mathrm{mM}$ Tris, pH 7.4 at $4{ }^{\circ} \mathrm{C}$, containing $0.32 \mathrm{M}$ sucrose and $3 \mathrm{mM} \mathrm{MgCl}$. Particulate (membrane) and cytosolic fractions were prepared by centrifugation at $100,000 \mathrm{~g}$ for $60 \mathrm{~min}$. The particulate fraction was resuspended in $40 \mathrm{mM}$ Tris and solubilized with $1 \%$ Lubrol PX. Homogenate fractions were also treated with $1 \%$ Lubrol PX. Samples were frozen in liquid $\mathrm{N}_{2}$ and stored at $-70^{\circ} \mathrm{C}$ until CaM levels were measured using a radioimmunoassay (RIA) (New England Nuclear, Boston, MA). Samples were heated for 6 min before further dilution into RIA buffer and assay. The antigenicity of CaM increased with time of sample heating so that the time of heating was carefully controlled. In some assays, the CaM antiserum used was that developed in sheep by Dr. Michael $W^{2}{ }^{3} h^{39}$. Results were comparable with those of the NEN radioimmunoassay kit. Samples from SAL- and AMPH-treated rats within any group were always analyzed simultaneously to avoid interassay variability. Statistical significance was determined by one way analysis of variance (ANOVA) with posttest Bonferroni $t$-test analysis calculated using GraphPad Instat and by a two-tail Students $t$-test.

\section{Materials}

AMPH was purchased from The University of Michigan Laboratory of Animal Medicine. Calmodulin was purified from bovine testes by the method of Dedman et al. ${ }^{5}$. Donkey anti-sheep IgG, rabbit serum, rice starch, polyethylene glycol 8000 , Tween 20 , Triton $\mathrm{X}-100$, bovine serum albumin and Lubrol PX were purchased from Sigma Chemical Co. (St. Louis, MO).

\section{RESULTS}

CaM content in brain areas after 4 weeks of withdrawal from repeated intermittent $A M P H$

In these experiments, we investigatd both a change in CaM content elicited by repeated AMPH treatment and

\section{TABLE I}

CaM concentration in rat striatum and limbic forebrain after repeated amphetamine treatment: 4 week withdrawal

Female Sprague-Dawley rats were treated with escalating doses of AMPH and given a challenge with $1.0 \mathrm{mg} / \mathrm{kg} \mathrm{AMPH}$ as described in Roseboom et al. ${ }^{37}$. Statistical differences were determined by ANOVA using the program GraphPad. Striatum: $n=$ 5; Cytosol, $P<0.001$ as determined by ANOVA, ${ }^{*}$ AS is significantly different from SS and AA at $P<0.05$. Membranes: $P<$ 0.03 as significantly determined by ANOVA, * AS is different from AA at $P<0.05$. Limbic forebrain: $n=10$; Cytosol, $P<$ 0.02 as determined by ANOVA, ${ }^{*}$ AA is significantly different from SA at $P<0.05$. Membranes, $P<0.0002$ as determined by ANOVA, ${ }^{+}$AS is significantly different from SS, SA and AA at $P<0.01$.

\begin{tabular}{|c|c|c|c|}
\hline \multirow[t]{2}{*}{ Brain area } & \multirow{2}{*}{$\begin{array}{l}\text { Treatment } \\
\text { group }\end{array}$} & \multicolumn{2}{|c|}{ 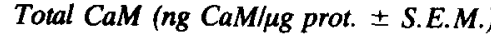 } \\
\hline & & Cyt & $M e m b$ \\
\hline \multirow{4}{*}{ Striatum } & SS & $0.45 \pm 0.04$ & $0.35 \pm 0.05$ \\
\hline & SA & $0.58 \pm 0.09$ & $0.30 \pm 0.02$ \\
\hline & AS & $0.72 \pm 0.09 *$ & $0.48 \pm 0.05 *$ \\
\hline & AA & $1.0 \pm 0.09^{*}$ & $0.31 \pm 0.04$ \\
\hline \multirow{4}{*}{$\begin{array}{l}\text { Limbic fore- } \\
\text { brain }\end{array}$} & SS & $0.62 \pm 0.05$ & $0.36 \pm 0.02$ \\
\hline & SA & $0.63 \pm 0.04$ & $0.34 \pm 0.02$ \\
\hline & AS & $0.71 \pm 0.06$ & $0.67 \pm 0.08^{+}$ \\
\hline & AA & $0.9 \pm 0.08^{*}$ & $0.39 \pm 0.04^{+}$ \\
\hline
\end{tabular}


the ability of a challenge injection of acute AMPH to alter subcellular CaM localization in both SAL and AMPH pretreatment groups. In the striatum, there was a significant increase in CaM in both the cytosol and membrane fractions after treatment with escalating doses of AMPH (AMPH-SAL group). This increase was reflected in both the concentration of CaM (Table I) and in total CaM (Fig. 1A). In striata from both SAL- and AMPH-treated groups, there was a nearly equal distribution of $\mathrm{CaM}$ in membranes and cytosol (Fig. 1A). Challenge with a low dose $(1 \mathrm{mg} / \mathrm{kg})$ of $\mathrm{AMPH}$, however, elicited a redistribution of the CaM in the AMPH-treated group, decreasing the $\mathrm{CaM}$ in the membranes and increasing the CaM in the cytosol. This redistribution is reflectcd as a significant decrease in the membrane:cytosol $(\mathrm{M} / \mathrm{C})$ ratio of
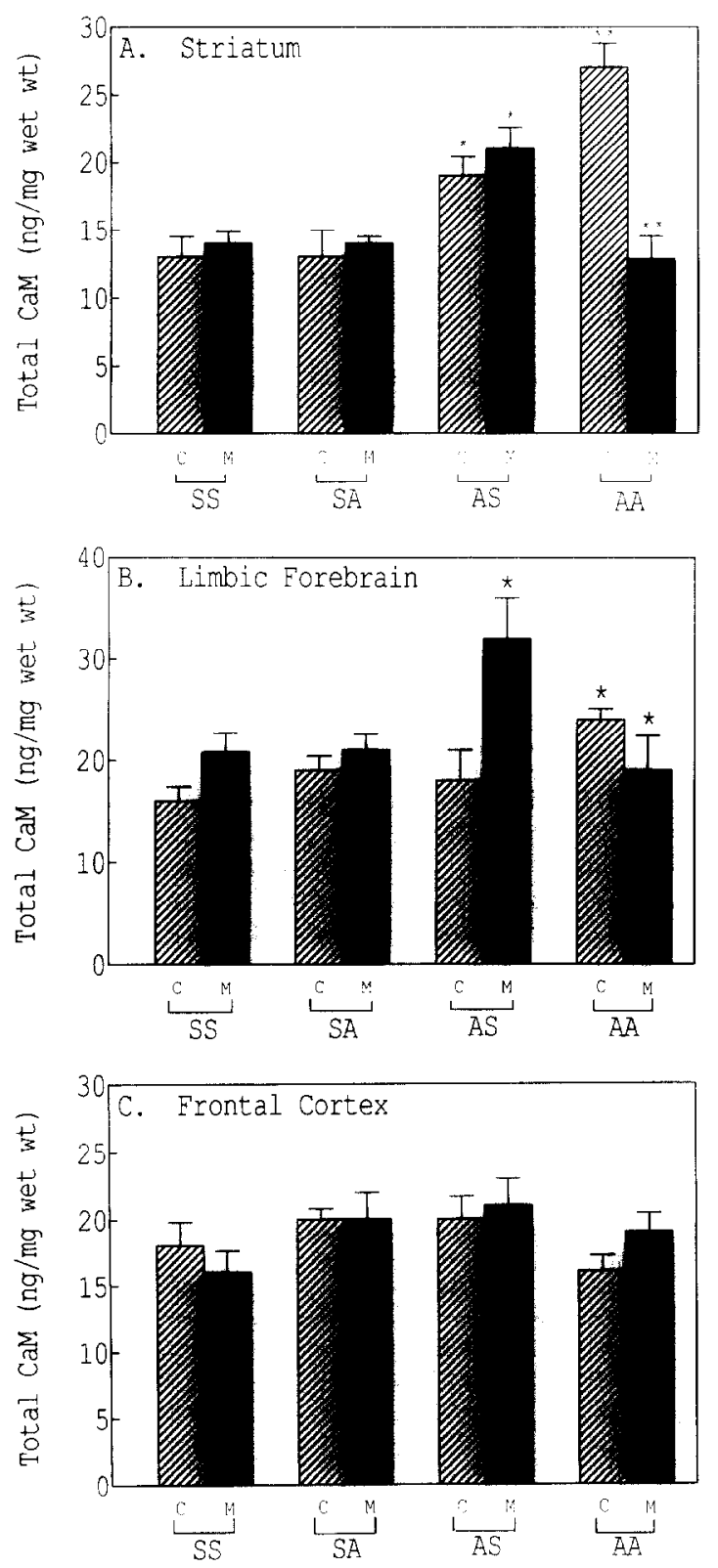

Fig. 1A-C. For legend see next caption.
CaM as shown in Table II. No change in the content or distribution of the CaM occurred with AMPH challenge in the SAL-treated animals.

A significant increase in CaM was evident in the membrane fraction of the mesolimbic area (nucleus accumbens + olfactory tubercle) after repeated AMPH treatment (Fig. 1B; Table I). In contrast to the striatum, there was no change in CaM in the mesolimbic cytosol fraction in the AMPH-SAL group. In the mesolimbic area, however, just as in the striatum, a challenge dose of $1 \mathrm{mg} / \mathrm{kg}$ AMPH decreased membrane-bound CaM and increased cytosolic CaM in the repeated AMPHtreated rats. The $\mathrm{M} / \mathrm{C}$ ratio in the limbic forebrain for
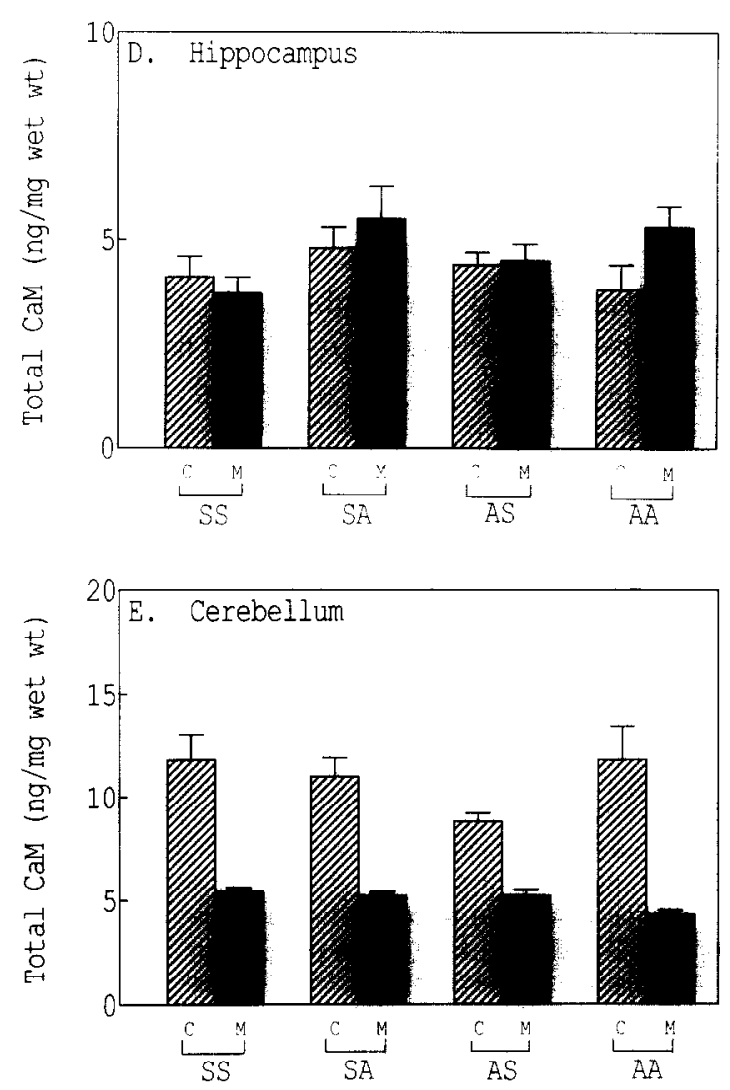

Fig. 1. CaM content in cytosol (C) and membrane (M) fractions from various rat brain areas after treatment with saline (S) or escalating doses of AMPH (A) and withdrawn 4 weeks. Female Sprague-Dawley rats were treated with SAL or escalating doses of AMPH and given a challenge dose of SAL or AMPH $30 \mathrm{~min}$ before sacrifice as described in Materials and Methods. Results are given as ng CaM per $\mathrm{mg}$ tissue wet weight \pm S.E.M. Statistical differences were determined by one-way ANOVA using postanalysis Bonferroni $t$-tests. A: striatum: $n=5$; cytosol, $P<0.0001$ as determined by ANOVA; AA is different from values for SS and AS at $P<0.05$, and for SA at $P<0.001$. Membranes: $P<0.002$ as determined by ANOVA; AS is different from SS at $P<0.05$ and from AA at $P<0.01$. B: limbic forebrain: $n=10$; cytosol, $P<$ 0.02 as determined by ANOVA; AA is significantly different from AS and SS at $P<0.05$. Membranes, $P<0.02$ as determined by ANOVA, AS is significantly different from SS and AA at $P<0.05$. C: frontal cortex: $n=5$. D: hippocampus: $n=5$. E: cerebellum: $n=5$. 


\section{TABLE II}

Effect of AMPH challenge on the subcellular localization of CaM after repeated AMPH: 4 weeks withdrawal

Female Sprague-Dawley rats were treated as described in the legend to Fig. 1. Membrane/Cytosol (M/C) ratios were calculated from values for total $\mathrm{CaM}$ in membrane and cytosol fractions as given in Fig. 1A-E.

\begin{tabular}{|c|c|c|c|c|}
\hline \multirow[t]{2}{*}{ Brain area } & \multicolumn{4}{|l|}{$M / C$ ratio } \\
\hline & $S S$ & $S A$ & $A S$ & $A A$ \\
\hline Striatum & $1.2 \pm 0.1$ & $1.1 \pm 0.2$ & $1.1 \pm 0.2$ & $0.5 \pm 0.06^{*}$ \\
\hline Limbic forebrain & $1.3 \pm 0.07$ & $1.1 \pm 0.1$ & $1.8 \pm 0.3$ & $0.8 \pm 0.15^{* *}$ \\
\hline Frontal cortex & $0.9 \pm 0.1$ & $0.97 \pm 0.1$ & $1.2 \pm 0.1$ & $1.2 \pm 0.1$ \\
\hline Hippocampus & $0.9 \pm 0.08$ & $1.2 \pm 0.1$ & $1.0 \pm 0.1$ & $1.3 \pm 0.2$ \\
\hline Cerebellum & $0.45 \pm 0.06$ & $0.45 \pm 0.04$ & $0.57 \pm 0.06$ & $0.4 \pm 0.04$ \\
\hline
\end{tabular}

* $P<0.007$ as determined by ANOVA, value for AA is significantly less than value for AS, SS or SA at $P<0.05$.

** $P<0.001$ as determined by ANOVA, value for AA is significantly less than value for AA, SS or SA

the AMPH-AMPH group was significantly less than that of the AMPH-SAL group (Table II). The challenge dose of AMPH had no effect on mesolimbic CaM content or distribution in the SAL-treated group.

No significant changes in CaM were evident in either membrane or cytosol fractions in medial prefrontal cortex, hippocampus or cerebellum after repeated AMPH treatment (Fig. 1C-E). There-was no change in CaM when the data were expressed as concentration in $\mathrm{ng} / \mu \mathrm{g}$ protein (data not shown). In addition, challenge with 1 $\mathrm{mg} / \mathrm{kg}$ AMPH did not change the subcellular distribution of CaM in either repeated saline- or AMPH-treated rats in these areas (Fig. 1C-E). In all brain areas, $\mathrm{CaM}$ was

\section{TABLE III}

CaM content in rat brain areas after repeated amphetamine treatment: no withdrawal

Female Sprague-Dawley rats were treated with escalating doses of AMPH or SAL as a control as described in the legend to Table I. Animals were sacrificed $\mathbf{3 0} \mathrm{min}$ after the last injection so that they were not withdrawn from the drug. Statistical differences were determined by a two-tail Student's $t$-test, $n=5$.

\begin{tabular}{llcc}
\hline Brain area & $\begin{array}{l}\text { Treatment } \\
\text { group }\end{array}$ & \multicolumn{2}{c}{ Total CaM (ng/mg wet wt) } \\
\cline { 3 - 4 } & & Cytosol & Membrane \\
\hline Striatum & SAL & $21.0 \pm 2.8$ & $21.0 \pm 3.7$ \\
& AMPH & $13.8 \pm 2.4$ & $12 \pm 1.6^{*}$ \\
Limbic forebrain & SAL & $18 \pm 1$ & $24 \pm 2.7$ \\
& AMPH & $14 \pm 1.3$ & $18 \pm 1.3$ \\
Frontal cortex & SAL & $12.8 \pm 1.6$ & $7.8 \pm 1.2$ \\
& AMPH & $7.4 \pm 1$ & $9.6 \pm 1.2$ \\
Hippocampus & SAL & $11 \pm 1.4$ & $8.0 \pm 0.4$ \\
& AMPH & $5.6 \pm 0.7^{* *}$ & $9.4 \pm 0.5$ \\
\hline
\end{tabular}

* $P<0.05$ as compared to corresponding value for SAL-treated rats; $* * P<0.01$ as compared to corresponding value for SALtreated rats. nearly equally distributed between membrane and cytosol fractions except in the cerebellum. In cerebellum, approximately $70 \%$ of the total CaM was located in the cytosol.

CaM content in various brain areas of rats not withdrawn from AMPH treatment

To determine whether the increase in CaM found in striatum and limbic forebrain developed during treatment with AMPH or required time after treatment, a group of rats was treated with the escalating doses of AMPH but sacrificed 30 min after the last injection. The results are shown in Table III. In contrast to the results from the withdrawn group, no increase in $\mathrm{CaM}$ in either membrane or cytosol fractions was detected in striatum after repeated AMPH treatment in the non-withdrawn group. In fact, there was a slight decrease in $\mathrm{CaM}$ in the striatal fractions from the AMPH-treated rats but it was not statistically significant. Similarly, there were no significant changes in $\mathrm{CaM}$ in the cytosol or membrane fractions in the mesolimbic area.

Significant decreases in CaM in the cytosol were found in the medial prefrontal cortex and the hippocampus from AMPH-treated rats as compared to controls. Decreases in CaM in the cytosol were not accounted for by increases in the membrane fractions.

\section{DISCUSSION}

In non-human animals, behavioral sensitization to AMPH is manifested as an increase in psychomotor stimulant effects of AMPH for long periods of time following drug treatment ${ }^{33}$. Behavioral sensitization in rats has several well-documented characteristics which include: (1) development with time after a single injection, (2) long-lasting effects, (3) requirement for intermittent but not continuous administration of AMPH and (4) increased degree of sensitization with time after withdrawal $^{32,33}$. Further, male rats show less robust sensitization than females. Therefore, in order for a neurochemical effect to be causally related to behavioral sensitization, it should demonstrate similar characteristics. An enhancement of releasability of DA has been demonstrated after repeated AMPH treatment and the effect correlates with the characteristics given above ${ }^{32,33}$. Enhanced release of DA has been demonstrated in striatum $^{19,33,34,41}$ and nucleus accumbens ${ }^{18,20,36}$ after repeated AMPH treatment. The increase in DA release can be demonstrated using AMPH, $\mathrm{K}^{+}$or electrical stimulation $^{2}$.

Previous studies with agents that alter dopaminergic sensitivity such as antipsychotic drugs, 6-hydroxydopamine and stimulant drugs, have suggested a relationship 
between CaM content or localization and dopaminergic activity in rat striatum ${ }^{8}$. We have previously reported that repeated AMPH treatment elicited an increase in $\mathrm{CaM}$ in rat striatum ${ }^{31,37}$. The increase was evident after a 5-day treatment with $3 \mathrm{mg} / \mathrm{kg}$ AMPH with 10-day withdrawal ${ }^{31}$ or a 4 -week treatment with escalating doses of $\mathrm{AMPH}^{37}$ as used in this study. We chose the escalating dose paradigm in our study because this treatment reduces the amount of individual variation in behavior measured after AMPH treatment in comparison with other treatments of constant low-dose regimens ${ }^{36}$. The time course of behaviors during treatment and after withdrawal as well as neurochemical changes in DA release, content and metabolite concentrations are welldocumented $^{32}$. In addition, this type of regimen is most likcly to be followed by humans taking the drug.

In this study we found that CaM was increased in subcellular fractions in both striatum and limbic forebrain after 4 weeks of withdrawal from an escalating dose regimen. A change in both areas would be expected if the increase in CaM were related to sensitization since both locomotor activity and stereotyped behaviors induced by AMPH are enhanced after repeated AMPH treatment. These behaviors are considered to be due to release of DA in the nucleus accumbens and caudate nucleus, respectively ${ }^{3,17,26}$. It is unclear whether dopaminergic systems in other brain areas such as frontal cortex or hippocampus are altered by the repeated AMPH treatment or play a significant role in the sensitization process. Robinson et al. ${ }^{34}$ found an enhancement in prefrontal cortex DA utilization in ovariectomized female rats that had been treated repeatedly with AMPH. There was no change in CaM in any of the fractions in the frontal cortex, hippocampus or cerebellum. Therefore, the principal change in CaM content after withdrawal from escalating doses of AMPH was found in areas of rat brain that are primarily involved in the behavioral sensitization.

Increases in CaM in rat striatum after chronic AMPH treatment have also been reported by Popov et al. ${ }^{30}$ and Popov and Matthies ${ }^{29}$. These investigators found increases in CaM after repeated AMPH were reflected in either cytosol or membrane fraction of striatum depending upon the dose. They reported an increase in CaM in hippocampus but not nucleus accumbens after a 21-day treatment with $5 \mathrm{mg} / \mathrm{kg}$ AMPH. The differences in our results could be due to the fact that (1) they used male rats, whereas we used female, and (2) their measurements were made after only two days of withdrawal or (3) a different treatment regimen was used.

In this study, no significant increase in CaM was observed in the striatum or mesolimbic areas in AMPHtreated rats that were not withdrawn from the drug. Fol- lowing the escalating dose regimen, sensitized responses to locomotor or stereotyped behavior do not develop until two weeks after completion of the escalating dose regimen $^{28}$. Interestingly, we found a significant decrease in $\mathrm{CaM}$ in the cytosol fractions from frontal cortex and hippocampus after repeated AMPH treatment and a trend toward a decrease in striatal fractions. Immediately after withdrawal from the escalating dose paradigm, rats show tolerance to the psychomotor effects of AMPH. The decrease in CaM could likely be related to the tolerance that has developed to the drugs. Since AMPH was in the rat brains at time of sacrifice, the decrease could be due to an acute effect of the AMPH. In preliminary studies we found no change in CaM content in striatum 30 min after acute doses of AMPH up to 8 $\mathrm{mg} / \mathrm{kg}$ (Gnegy, unpublished studies). The reduction of CaM after repeated AMPH seems not to involve a translocation of CaM since a corresponding increase in another fraction was not found. We have found that the CaM antibody obtained both from Dr. Welsh and from the RIA kit will measure CaM bound to other proteins. l'herefore a reduction in detection of CaM due to increased binding of $\mathrm{CaM}$ to $\mathrm{CaM}$-binding proteins cannot account for the results. It is possible that AMPH treatment could alter the turnover of CaM in brains areas.

We demonstrated previously that a challenge with a lower dose of AMPH would alter the localization of $\mathrm{CaM}$ in striatum from rats treated with escalating doses of $\mathrm{AMPH}^{37}$. A low dose of AMPH, $1 \mathrm{mg} / \mathrm{kg}$, had no effect on CaM lozalization in striatum from SAL-treated rats, but did elicit a membrane-to-cytosol translocation of CaM in AMPH-treated rats. Higher doses of AMPH, however, have been found to cause a membrane-to-cytosol translocation of CaM in control, non-AMPHtreated rats ${ }^{13}$, although Popov and Matthies ${ }^{29}$ found that $5 \mathrm{mg} / \mathrm{kg}$ AMPH caused a translocation of CaM from cytosol to membrane in rat striatum. Our results suggest that the system responsible for the translocation showed greater activity after the repeated AMPH treatment due either to enhanced postsynaptic responsiveness or an increased DA release. Both $\mathrm{AMPH}^{13}$ and $\mathrm{DA}^{12,25}$ have been shown to elicit the translocation of CaM from membranes to cylosol using striatal membrane fractions or slices. A role for the $\mathrm{D}_{1} \mathrm{DA}$ receptor in this activity was suggested by the fact that cAMP itself could elicit a membrane-to-cytosol translocation of CaM in vitro ${ }^{9,12}$. The translocation of CaM from membranes to cytosol correlated with a decreased responsiveness of adenylyl cyclase to $\mathrm{DA}^{25}$. Similarly, we found that challenge with low-dose AMPH induced a pronounced down-regulation of DA-stimulated adenylyl cyclase activity in rats withdrawn 2-4 weeks from the escalating dose of AMPH regimen ${ }^{37}$. Thus the AMPH-elicited translocation of 
CaM has functional consequences for the cell as demonstrated by adenylyl cyclase activity and may profoundly affect other systems as well. Increasing the concentration of CaM in the cytosol could activate cytosolic CaM-dependent enzymes such as CaM-dependent protein kinases, CaM-dependent phosphodiesterases or calcineurin.

The function of the increase in CaM after withdrawal from repeated AMPH is not known but the level of involvement of $\mathrm{CaM}$ in many areas of synaptic function suggest that it could play a role in either the increased release of DA or heightened postsynaptic responses, or both. CaM has been shown to have a role in increasing neurotransmitter release ${ }^{6,23}$ in particular through phosphorylation of synapsin 1 by CaM-dependent protein kinase $\mathrm{II}^{23,27}$. Our laboratory has demonstrated that $\mathrm{CaM}$ can increase the sensitivity and response of striatal adenylyl cyclase to DA by a potentiative interaction with $\mathrm{G}_{\mathrm{s}}{ }^{11,14,15}$. We found a small yet significant increase in DA-stimulated adenylyl cyclase activity 4 weeks after withdrawal from treatment with escalating doses of $\mathrm{AMPH}^{37}$.

\section{REFERENCES}

1 Beninger, R.J. and Hahn, B.L., Pimozide blocks establishment but not expression of amphetamine-produced environment-specific conditioning, Science, 220 (1983) 1304-1306.

2 Casteñada, E., Becker, J. and Robinson, T.E., The long-term effects of repeated amphetamine treatment in vivo on amphetamine, $\mathrm{KCl}$ and electrical stimulation evoked striatal dopamine release in vitro, Life Sci., 42 (1988) 2447-2456.

3 Costall, B. and Naylor, R.J., Mesolimbic and expyramidal sites for the mediation of stereotyped behavior patterns and hyperactivity by amphetamine and apomorphine in the rat. In E.H. Ellinwood and M.M. Kilbey (Eds.), Cocaine and Other Stimulants, Plenum, New York, 1977, pp. 47-76.

4 Davis, J.M. and Schlemmer, R.F., The amphetamine psychosis. In J. Caldwell (Ed.), Amphetamines and Related Stimulants, CRC Press, Boca Raton, FL, 1980, pp. 161-173.

5 Dedman, J.D., Potter, J.D., Jackson, R.L. and Means, A.R., Physicochemical properties of rat testis $\mathrm{Ca}^{2+}$-dependent regulator protein of cyclic nucleotide phosphodiesterase. Relationship of $\mathrm{Ca}^{2+}$ binding, conformational changes, and phosphodiesterase activity, J. Biol. Chem., 252 (1977) 8415-8422.

6 DeLorenzo, R.J., Role of calmodulin in neurotransmitter release and synaptic function, Ann. N.Y. Acad. Sci., 256 (1980) 92-109.

7 Ellinwood, E.H., Amphetamine psychosis: individuals, settings, and sequences. In E.H. Ellinwood and S. Cohen (Eds.), Current Concepts on Amphetamine Abuse, U.S. Government Printing Office, Washington, D.C., 1972, pp. 143-157.

8 Gnegy, M.E., Relationship of calmodulin and dopaminergic activity in the striatum, Fed. Proc., 41 (1982) 51-55.

9 Gnegy, M.E., Costa, E. and Uzunov, P., Regulation of transsynaptically elicited increase of $3^{\prime}: 5^{\prime}$-cyclic AMP by endogenous phosphodiesterase activator, Proc. Natl. Acad. Sci. U.S.A., 73 (1976) 352-355.

10 Gnegy, M.E., Nathanson, J.A. and Uzunov, P., Release of the phosphodiesterase activator by cyclic AMP-dependent ATP:protein phosphotransferase from subcellular fractions of rat brain, Biochim. Biophys. Acta, 497 (1977) 75-85.
In summary, changes in CaM content in rat brain after an escalating dose regimen of AMPH show some correlation with characteristics of behavioral sensitization which develop after this treatment. The increase in $\mathrm{CaM}$ was found in striatum and limbic forebrain, areas involved in AMPH-induced stereotyped and locomotor behaviors, respectively. Heightened stimulus-induced DA release has been measured in these areas ${ }^{32}$ in AMPH-sensitized rats. The increase in CaM is long-lasting, i.e., it is evident 4 weeks after ceasing the AMPH treatments and develops with time after withdrawal from the drug. In rats not withdrawn from AMPH, CaM levels either did not change from controls or were decreased. Therefore changes in CaM could contribute to the neurochemical changes underlying sensitized behaviors evident after repeated AMPH treatment.

Acknowledgements. The authors are grateful to Dr. Terry Robinson (Department of Psychology, University of Michigan) for his helpful discussions over the course of this study. This work was supported by Grant DA05066 from the National Institutes for Drug Abuse.

11 Gnegy, M.E. and Treisman, G., Effect of calmodulin on dopamine-sensitive adenylate cyclase activity in rat striatal membranes, Mol. Pharmacol., 19 (1981) 256-263.

12 Gnegy, M.E., Uzunov, P. and Costa, E., Regulation of the dopamine stimulation of striatal adenylate cyclase by an endogenous $\mathrm{Ca}^{2+}$-binding protein, Proc. Natl. Acad. Sci. U.S.A., 73 (1976) 3887-3890.

13 Hanbauer, I., Pradham, S. and Yang, H.-Y.T., Role of calmodulin in dopaminergic transmission, Ann. N.Y. Acad. Sci., 356 (1980) 292-303.

14 Harrison, J.K., Hewlett, G.H.K. and Gnegy, M.E., Regulation of calmodulin-sensitive adenylate cyclase by the stimulatory G-protein, Gs*, J. Biol. Chem., 264 (1989) 15880-15885.

15 Harrison, J.K., Mickevicius, C.K. and Gnegy, M.E., Differential regulation by calmodulin of basal, GTP- and dopaminestimulated adenylate cyclase activities in bovine striatum, $J$. Neurochem., 51 (1988) 345-352.

16 Heffner, T.G., Hartman, J.A. and Seiden, L.S., A rapid method for the regional dissection of the rat brain, Pharmacol. Biochem. Behav., 13 (1980) 453-456.

17 Iversen, S.D., Brain dopamine systems and behavior. In L.L. Iversen, S.D. Iversen and S.H. Snyder (Eds.), Handbook of Psychopharmacology, Plenum, New York, 1977, pp. 333-383.

18 Kazahaya, Y., Akimoto, K. and Otsuki, S., Subchronic methamphetamine treatment enhances methamphetamine- or cocaineinduced dopamine efflux in vivo, Biol. Psychiatry, 25 (1989) 903-912.

19 Kolta, M.B., Shreve, P., De Souza, V. and Uretsky. N.J., Time course of the development of the enhanced behavioral and biochemical responses to amphetamine after pretreatment with amphetamine, Neuropharmacology, 24 (1985) 823-829.

20 Kolta, M.G., Shreve, P. and Uretsky, N.J., Effect of pretreatment with amphetamine on the interaction between amphetamine and dopamine neurons in the neucleus accumbens, Neuropharmacology, 28 (1989) 9-14.

21 Kramer, J.C., Fischman, V.S. and Littlefield, D.C., Amphetamine abuse, J. Am. Med. Assoc., 201 (1967) 89-93.

22 Kuczenski, R. and Leith, N.J., Chronic amphetamine: is dopamine a link in or mediator of the development of tolerance and 
reverse tolerance? Pharm. Biochem. Behav., 15 (1981) 405-413.

23 Llinás, R., McGuiness, T.L., Leonard, C.S., Sugimori, M. and Greengard, P., Intraterminal injection of synapsin 1 or calcium/ calmodulin-dependent protein kinase 11 alters neurotransmitter release at the squid giant synapse, Proc. Natl. Acad. Sci. U.S.A., 82 (1985) 3035-3039.

24 Manalan, A.S. and Klee, C.B., Calmodulin, Adv. Cycl. Nucl. Prot. Phosphor. Res., 18 (1984) 227-278.

25 Memo, M., Lovenberg, W. and Hanbauer, I., Agonist-induced subsensitivity of adenylate cyclase coupled with a dopamine receptor in slices from rat corpus striatum, Proc. Natl. Acad. Sci. U.S.A., 79 (1982) 4456-4460.

26 Moore, K.E., Amphetamines: biochemical and behavioral actions in animals. In L.L. Iversen, S.D. Iversen and S.H. Snyder (Eds.), Handbook of Psychopharmacology, Plenum, New York, 1978, pp. 41-98.

27 Nichols, R.A., Sihra, T.S., Czernick, A.J., Nairn, A.C. and Greengard, P., Calcium/calmodulin-dependent protein kinase II increases glutamate and noradrenaline release from synaptosomes, Nature, 343 (1990) 647-651.

28 Paulson, P.E., Camp, D.M. and Robinson, T.E., The time course of transient behavioral depression and persistent behavioral sensitization in relation to regional brain monoamine concentrations during amphetamine withdrawal in rats, Psychopharmacology, 103 (1991) 480-492.

29 Popov, N. and Matthies, H., Influence of dopamine receptor agonists and antagonists on calmodulin translocation in different brain regions, Eur. J. Pharmacol., 172 (1989) 205-210.

30 Popov, N., Schulzeck, S., Nüss, D., Vopel, A.-U., Jendrny, C., Struy, H. and Matthies, H., Alterations in calmodulin content of rat brain areas after chronic application of haloperidol and amphetamine, Biomed. Biochim. Acta, 47 (1988) 435-441.

31 Roberts-Lewis, J.M., Welsh, M.J. and Gnegy, M.E., Chronic amphetamine treatment increases striatal calmodulin in rats, Brain Research, 384 (1986) 383-386.

32 Robinson, T.E., The neurobiology of amphetamine psychosis: evidence from studies with an animal model. In T. Nakazawa (Ed.), Taniguchi Symposia on Brain Sciences, Vol. 14, Biolog- ical Basis of Schizophrenic Disorders, Japan Scientific Societies Press, Tokyo, in press.

33 Robinson, T.E. and Becker, J.B., Enduring changes in brain and behavior produced by chronic amphetamine administration: a review and evaluation of animal models of amphetamine psy. chosis, Brain Res. Rev., 11 (1986) 157-198.

34 Robinson, T.E., Becker, J.B., Moore, C.J., Castenada, E. and Mittleman, G., Enduring enhancement in frontal cortex dopamine utilization in an animal model of amphetamine psychosis, Brain Research, 343 (1985) 374-377.

35 Robinson, T.E. and Camp, D.M., Long-lasting effects of escalating doses of $d$-amphetamine on brain monoamines, amphetamine-induced stereotyped behavior and spontaneous nocturnal locomotion, Pharmacol. Biochem. Behav., 26 (1987) 821-827.

36 Robinson, T.E., Jurson, P.A., Bennett, J.A. and Bentgen, K.M., Persistent sensitization of dopamine neurotransmission in ventral striatum (nucleus accumbens) produced by prior experience with $(+)$-amphetamine: a microdialysis study in freely moving rats, Brain Research, 462 (1988) 211-222.

37 Roseboom, P.H., Hewlett, G.H.K. and Gnegy, M.E., Repeated amphetamine administration alters the interaction between $D_{1}$. stimulated adenylyl cyclase activity and calmodulin in rat striatum, J. Pharmacol. Exp. Ther., 255 (1990) 197-203.

38 Sato, M., Acute exacerbation of methamphetamine psychosis and lasting dopaminergic supersensitivity - a clinical survey, Psychopharmacol. Bull., 22 (1986) 751-756.

39 Sweet, S.C., Gnegy, M.E. and Welsh, M.J., Presence of matrix-specific antibodies in affinity-purified polyclonal antibodies, J. Immunol. Methods, 136 (1990) 31-36.

40 Vezina, P. and Stewart, J., The effect of dopamine receptor blockade on the development of sensitization to the locomotor activating effects of amphetamine and morphine, Brain Research, 499 (1989) 108-120.

41 Yamada, S., Kojima, H., Yokoo, H., Tsutsumi, T., Takamuki, K., Anraku, S., Nishi, S. and Inanaga, K., Enhancement of dopamine release from striatal slices of rats that were subchronically treated with metamphetamine, Biol. Psychiatry, 24 (1988) 399-408. 